\title{
Factors influencing the quality of life in children after biliary atresia treatment
}

\author{
Yuanyuan Liang ${ }^{1,2} \wedge$, He Yu ${ }^{3}$, Fanke Shu ${ }^{1,2}$, Wenjiao Huang ${ }^{1,2}$, Xiaoping Jiang ${ }^{1}$, Zhicheng Xu ${ }^{1}$, \\ Ting Zhang ${ }^{1,2}$, Bo Xiang ${ }^{1}$, Shuguang Jin ${ }^{1}$ \\ ${ }^{1}$ Department of Pediatric Surgery, West China Hospital, Sichuan University, Chengdu, China; ${ }^{2}$ West China School of Nursing, Sichuan University, \\ Chengdu, China; ${ }^{3}$ West China School of Clinical Medicine, Sichuan University, Chengdu, China \\ Contributions: (I) Conception and design: Y Liang, S Jin; (II) Administrative support: B Xiang; (III) Provision of study materials or patients: H Yu, X \\ Jiang, Z Xu; (IV) Collection and assembly of data: W Huang, F Shu, T Zhang; (V) Data analysis and interpretation: Y Liang, S Jin; (VI) Manuscript \\ writing: All authors; (VII) Final approval of manuscript: All authors. \\ Correspondence to: Shuguang Jin. Department of Pediatric Surgery, West China Hospital, Sichuan University, No. 37 Guoxue Xiang, Chengdu, China. \\ Email: shgjin2003@aliyun.com.
}

Background: Despite surgical treatment, children with biliary atresia (BA) may face many problems which seriously affect their quality of life. However, there is a paucity of studies in China examining the quality of life of these children after BA treatment. This study investigated the current status of the quality of life of children after BA treatment and analyzed the influencing factors so as to provide a scientific basis for the development of targeted interventional measures.

Methods: From May 1 to May 31, 2021, the STAR questionnaire method was used to conduct crosssectional surveys in children following BA surgery in the West China Hospital of Sichuan University, China. The basic information questionnaire was used to investigate the general characteristics of the children. The children were also given quality of life questionnaires during follow-up sessions after surgical treatment for BA. In addition, a 12-point health survey was used to assess the physical and mental health of the children's caregivers. Age- and gender-matched healthy children were recruited as controls. Correlation analysis and multiple linear regression equations were established to explore the influencing factors of the quality of life of children after surgery for BA.

Results: After surgery, children with BA experienced significantly lower physical health, emotional function, social function, cognitive function, and quality of life compared to healthy children $(\mathrm{P}<0.050)$. Coexisting diseases had a statistically significant impact on the quality of life of children with BA $(b=-12.566$; $\mathrm{t}=-2.343 ; \mathrm{P}=0.021$ ), and the caregiver's understanding level of liver transplantation also had a statistically significant impact on the quality of life of these children $(b=6.481 ; t=2.376 ; \mathrm{P}=0.021)$.

Conclusions: The quality of life of children after BA surgery was significantly lower than that of agematched healthy children. Co-existing diseases and the caregiver's understanding level of liver transplantation were the main factors affecting the quality of life of these children.

Keywords: Biliary atresia (BA); children's quality of life questionnaire; 12-item health survey; quality of life; influencing factors

Submitted Aug 05, 2021. Accepted for publication Sep 15, 2021.

doi: $10.21037 /$ tp-21-391

View this article at: https://dx.doi.org/10.21037/tp-21-391

$\wedge$ ORCID: 0000-0002-8265-1645. 


\section{Introduction}

Biliary atresia (BA) is a serious congenital hepatobiliary disease characterized by dysfunction and occlusion of the intrahepatic and extrahepatic bile ducts and liver fibrosis, the pathogenesis is still unclear, and the current main point of view is that it is related to viral infection in the embryonic or perinatal period. The incidence of BA ranges from 1 in 5,000 to 1 in 12,000 (1), with a higher incidence in Japan and China compared to that in Europe and America. Without treatment, the average survival period is less than 1 year and approximately $75 \%$ of children will require liver transplantation for long-term survival (2). At present, the main form of treatment for children with BA is the Kasai operation. Advances in this technique and improved public cognition of the condition have seen the 2-year autologous liver survival rate approach 60\% (3). However, children with BA can still face many serious complications after surgery, including postoperative cholangitis, portal hypertension, variceal bleeding, biochemical abnormalities, hepatic osteodystrophy, growth problems, and problems associated with long-term use of high-dose hormones. All this can lead to a significant decline in the quality of life and serious financial burden. However, the few studies reporting the quality of life of BA children after surgery remain controversial. Some studies have reported that about $50 \%$ of children experienced a low quality of life after BA (4), especially in the 5-12 year age group (5). On the contrary, other reports have suggested that children with BA experience the same quality of life as healthy children in adulthood (6-8). This current study investigated the current quality of life of children after BA treatment and analyzed the influencing factors. We present the following article in accordance with the SURGE reporting checklist (available at https://dx.doi.org/10.21037/tp-21-391).

\section{Methods}

\section{Participants}

Children with BA who were diagnosed and underwent surgery in the West China Hospital of Sichuan University from May 1 to May 31, 2021, were recruited for this study. Age- and gender-matched healthy children were enrolled as the control group. The STAR questionnaire method was used to survey the children during follow-up consultations. Children were included in the study if the met the following inclusion criteria: (I) a confirmed BA diagnosis and all operations were completed in the West China Hospital of Sichuan University; (II) the quality of life questionnaire was administered 3 months or more after the completion of the surgery; (III) the questionnaire was completed with no conflicting content; and (IV) the caregiver spent $\geq 4$ hours/day caring for the child in the past month and voluntarily participates in this investigation. The following exclusion criteria were applied: (I) children presenting with coexisting diseases that seriously affect the quality of life, such as esophageal atresia, chromosomal abnormalities, cyanotic heart disease, and other organ dysfunctions; and (II) the caregiver cannot correctly understand and complete the questionnaire due to cultural, medical, or other reasons.

\section{Research tools}

Questionnaire for the basic characteristics of the child This form was designed in-house and includes the following basic information: the child's age, gender, place of residence, number of siblings, family plans to have another child, the child's feeding routine, co-existing diseases, method of surgery, and the child's age at the time of surgery.

Questions related to the child's family include the following: the marital status of the child's family, the type of family where the child lives, the main daily caregiver of the child, the education status of the main caregiver, the caregiver's understanding level of BA and liver transplantation, the monthly family income, the payment method for medical expenses, and the average monthly costs associated with the child post-surgery.

Questions related to post-surgical status include the following: duration of oral antibiotic administration postsurgery, regular follow-up visits to the hospital, presence or absence of cholangitis, when cholangitis occurs and whether it is complicated with other systemic infections, and the work conditions and emotional well-being of the caregivers post-surgery.

\section{Chinese Mandarin version of the Pediatric Quality of Life Inventory [TM] (PedsQL[TM]) 4.0 (9)}

The Pediatric Quality of Life Inventory scale was released in 2011 and is divided into a standard version and a shortterm version. For children over 5 years old, there is a self-filled version and a parent-filled version. This study used the standard version and the parent-filled version to investigate the quality of life of children up to 18 years old. There are four dimensions to this questionnaire: physical health, emotional function, social function, and cognitive function. The latter three dimensions collectively reflect 
the psychological and social well-being of the children. The number of items and behavior descriptions in each dimension differ slightly for different age groups. Each item is a survey of the frequency of an event in the most recent month. There are 5 possible answers ranging from 0 to 4 , with 0 representing never, 1 representing almost never, 2 representing sometimes, 3 representing often, and 4 representing almost always. These scores are then converted to 100 points, 75 points, 50 points, 25 points, and 0 points, respectively. The total score for each dimension is the sum of the scores of the answered items divided by the full marks of these items. The scores of each dimension range from 0 to 100 . The higher the score, the better the quality of life. The use of the PedsQL[TM] scale has been authorized by Professor JW Varni and the translated Chinese version has been verified by the issuing agency.

\section{The 12-item short form health survey (SF-12)}

The 12-item short form health survey (SF-12) $(10,11)$ is an abbreviated form of the 36-item health survey with the advantages of being concise, easy-to-understand, and quick to fill in. SF-12 has been widely used in different countries and regions to assess health-related quality of life. The SF-12 scale has 12 items and 8 dimensions for assessing health-related quality of life, including physical functioning $(\mathrm{PF})$, role-physical (RP), body pain (BP), general health (GH), vitality (VT), social functioning (SF), role-emotional (RE), and mental health (MH). Among them, PF, RP, BP and $\mathrm{GH}$ are included in the physical component summary (PCS), while VT, SF, RE, and MH are included in the mental component summary (MCS). The higher the score, the better the health-related quality of life.

\section{Research methods}

This study adopted a cross-sectional survey method and the STAR questionnaire method was used. Surveys were carried out in the follow-up group of BA children in West China Hospital of Sichuan University. The basic information questionnaire was used to collate the general data of the children, the PedsQL[TM] was used to assess the quality of life of children after BA, and the SF-12 scale was used to evaluate the physical and mental health of the caregivers of the children. Information regarding the purpose, meaning, medical ethics, confidentiality, and voluntary nature of the questionnaire, as well as the filling requirements and precautions were provided to the participants prior to completion of the survey. After the questionnaire was completed, it was verified by 3 investigators, and a presurvey was conducted among the family members of the children, and the questionnaire was released after confirmation. There were 6 investigators in the followup group, including 1 associate professor, 2 attending physicians, 1 pharmacist, 1 nurse-in-charge, and 1 nurse. All investigators have been uniformly trained. Data export, sorting, and analysis was verified by two independent researchers.

\section{Sample size}

According to the rough estimation method of sample content proposed by Kendall, the sample size is generally determined as 10-20 times the survey index (12). In this study, the PedsQL[TM] scale has 4 dimension items, and SF-12 has 8 dimension items, that is, 80 examples around the sample.

\section{Statistical analysis}

The SPSS 26.0 software was used for data processing and statistical analysis. Normally distributed measurement data is represented by mean \pm standard deviation $(\bar{x} \pm s)$, nonnormally distributed measurement data is represented by median (interquartile range) [M (IQR)], and count data is represented by frequency and composition ratio. The bivariate rank correlation analysis method was used for single-factor correlation analysis, and the variables with statistical differences in the single-factor correlation analysis were included as independent variables to construct a multifactor linear regression equation. A two-sided test was used, and the test level was set to $\alpha=0.05$.

\section{Etbical principles}

All procedures performed in this study involving human participants were in accordance with the Declaration of Helsinki (as revised in 2013). This study has been reviewed and approved by the Biomedical Ethics Committee of the West China Hospital, Sichuan University, China (approval number: \#20191082) and informed consent was taken from parents of all the patients.

\section{Results}

\section{Inclusion of research subjects}

Between May 1 to May 31, 2021, 79 questionnaires were 
Table 1 Comparison of quality of life scores between children with biliary atresia and age-matched healthy children (points)

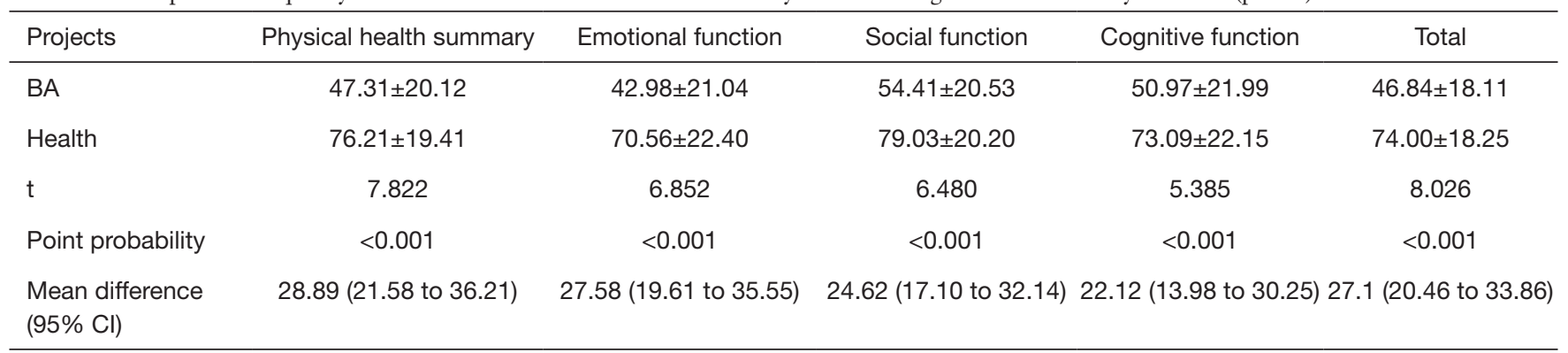

BA, biliary atresia; $\mathrm{Cl}$, confidence interval.

received. A total of 10 questionnaires were excluded, including 4 repeated questionnaires, 2 questionnaires with incorrect information, and 4 mismatches in filling in the 3 scales. A total of 70 valid questionnaires (88.61\%) from 70 children were enrolled, including 31 males and 39 females, with an average age of 11 months (IQR, 34.5 months). A total of 49 questionnaires were collected from age- and gender-matched children. The average age of children in the normal group was 11 months (IQR, 32.9 months), with 22 males and 27 females. The negative emotions of the caregivers were mainly manifested as fatigue $(61.4 \%)$ and depression (44.3\%), while $11.4 \%$ felt unbalanced, $20.0 \%$ felt lonely, and $27.1 \%$ felt that they were easy to anger. Unfortunately, only $27.1 \%$ of caregivers did not report any particular feelings.

\section{A comparison of the quality of life scores between $B A$ children and age-matched bealthy children}

Following surgery, children with BA had significantly lower scores for physical health, emotional function, social function, cognitive function, and quality of life compared to age-matched healthy children $(\mathrm{P}<0.050$; Table 1$)$.

\section{Univariate analysis of the factors affecting the quality of life in $B A$ children after treatment}

The quality of life of BA children post-surgery was significantly correlated with co-existing diseases $(r=-0.277$; $\mathrm{P}=0.020)$, understanding level of liver transplantation $(\mathrm{r}=0.254 ; \mathrm{P}=0.034)$, the payment method of medical expenses $(r=-0.275 ; P=0.021)$, the average monthly medical expenses post-surgery $(\mathrm{r}=-0.301 ; \mathrm{P}=0.011)$, and the duration of oral antibiotics administration $(\mathrm{r}=0.358 ; \mathrm{P}=0.002)$. See Table 2 for details.

\section{Multiple linear regression analysis of the quality of life of children after BA treatment}

Taking the total score of the physical health of the children after BA as the dependent variable, and statistically significant variables in single-factor correlation analysis were included as independent variables to construct a multivariate linear regression equation. The results showed that coexisting diseases had a statistically significant impact on the quality of life of children with BA $(b=-12.566 ; t=-2.343$; $\mathrm{P}=0.021$ ). Furthermore, the caregiver's understanding level of liver transplantation also exerted a significant impact on the quality of life of BA children after surgery $(b=6.481$; $\mathrm{t}=2.376 ; \mathrm{P}=0.021$ ). The model fit (adjusted $\mathrm{R}^{2}$ value) was 0.208. The hypothesis test result of the regression model showed $\mathrm{F}=4.630$ and $\mathrm{P}=0.001$ (Table 3).

\section{Discussion}

Health-related quality of life (HRQoL) is an important reference index for providing long-term comprehensive care for children with chronic diseases, and can enable medical workers to better understand the health status of such children $(13,14)$. Problems related to the quality of life have gradually become the "hidden pathology" of chronic diseases such as BA, but there are few studies on the quality of life of children with BA, especially in China. Consistent with the findings of Sundaram et al. (5), this study confirmed that the scores of all dimensions of quality of life were significantly lower in BA children post-surgery compared to those of healthy children. Furthermore, when children with BA have co-existing diseases, their quality of life will further decline, and thus developing management and support strategies for these patients is crucial.

Kasai portojejunostomy is currently the preferred treatment for BA (15) to relieve cholestasis and inflammation 
Table 2 The univariate correlation analyses of the factors affecting the quality of life of children with biliary atresia

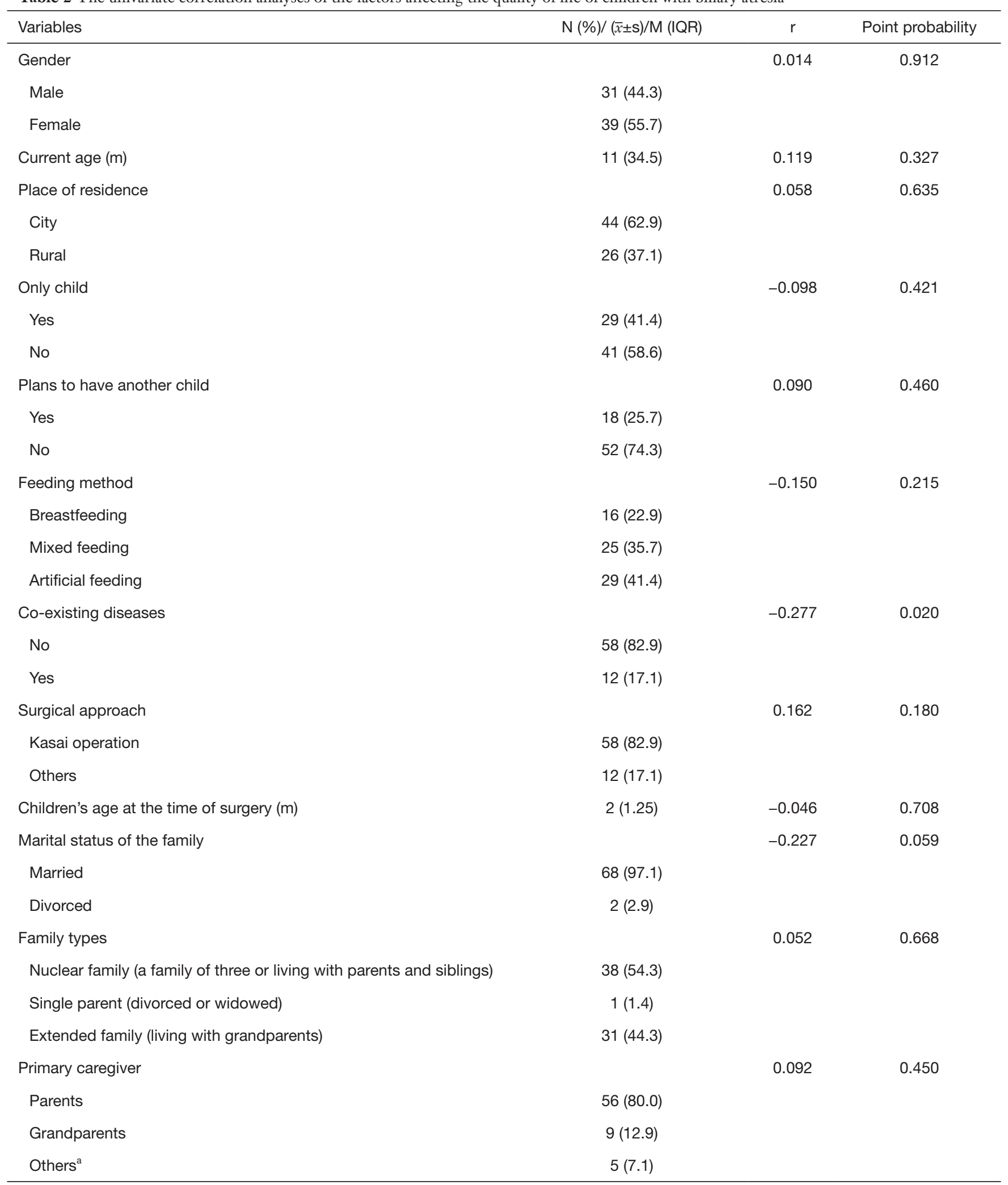

Table 2 (continued) 
Table 2 (continued)

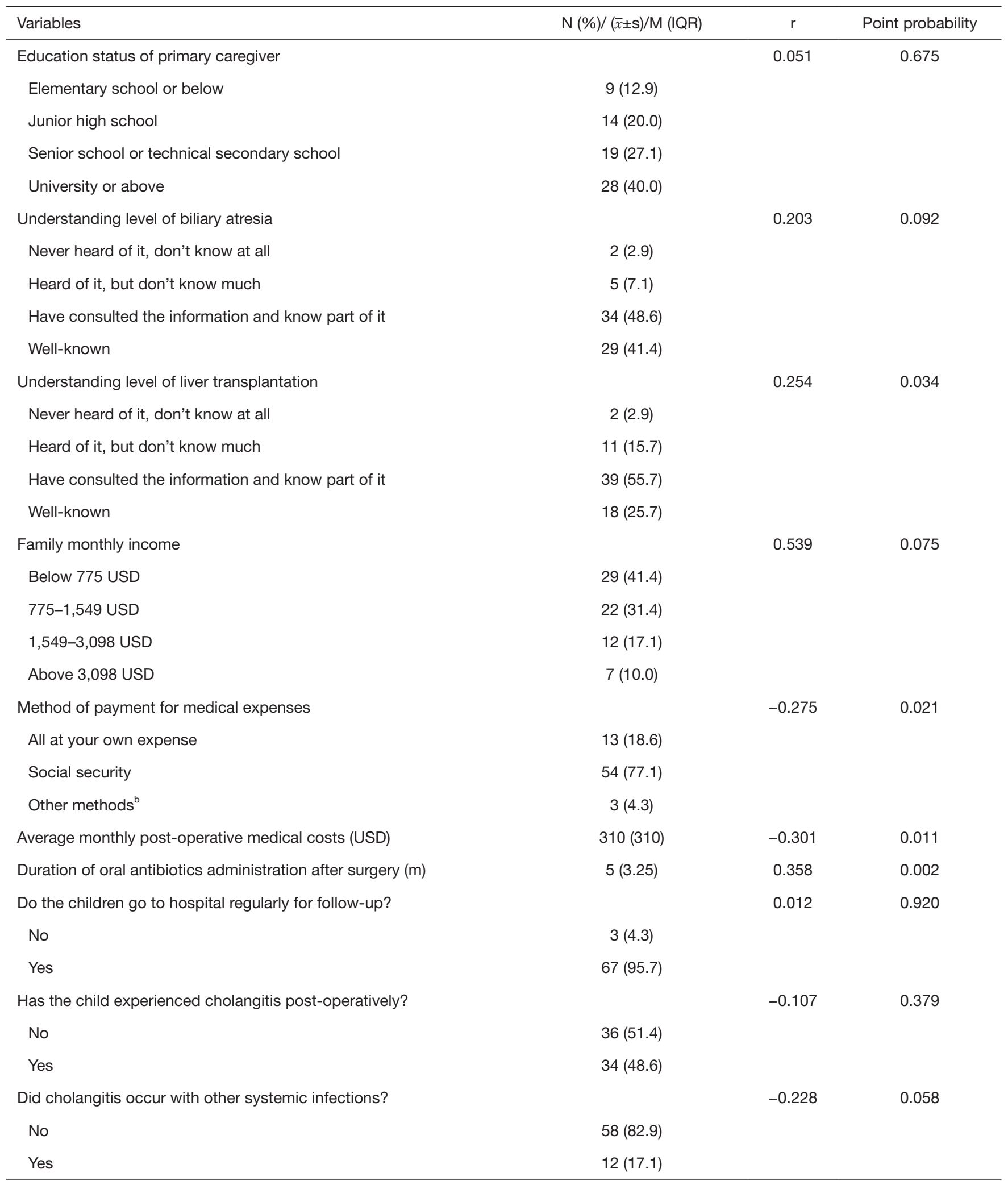

Table 2 (continued) 
Table 2 (continued)

\begin{tabular}{|c|c|c|c|}
\hline Variables & $\mathrm{N}(\%) /(\bar{x} \pm \mathrm{s}) / \mathrm{M}(\mathrm{IQR})$ & r & Point probability \\
\hline Full time job & $13(18.6)$ & & \\
\hline Half-work and half-rest & $13(18.6)$ & & \\
\hline No job & $44(62.9)$ & & \\
\hline Psychological health of caregiver after the child's surgery (MCS) & $44.81 \pm 7.81$ & 0.223 & 0.064 \\
\hline
\end{tabular}

${ }^{a}$, other options for the main caregiver of the child's daily life refer to the combination of parents and (grand) parents or the combination of nanny and other people; ${ }^{b}$, other methods of payment of medical expenses refer to hospitals or poverty subsidies and a combination of methods including own expense. IQR, interquartile range; m, months; USD, United States Dollar; PCS, physical component summary; MCS, mental component summary.

Table 3 Multiple linear regression analysis of the quality of life of children after biliary atresia

\begin{tabular}{lccccc}
\hline Variables & $\mathrm{b}$ & Std. Error & Sig. & $\mathrm{P}$ & \multicolumn{1}{c}{$95 \% \mathrm{Cl}$ for b } \\
\hline Co-existing diseases $^{\mathrm{a}}$ & -12.566 & 5.317 & -2.343 & 0.021 & -23.189 to -1.943 \\
Understanding level of liver transplants & 6.481 & 2.728 & 2.376 & 0.021 & 1.031 to 11.930 \\
Method of payment for medical expenses & -3.668 & 2.237 & -1.640 & 0.106 & -8.136 to 0.800 \\
Average monthly post-operative medical costs & -0.001 & 0.000 & -1.799 & 0.077 & -0.001 to 0.000 \\
Duration of oral antibiotics administration after surgery $(\mathrm{m})$ & 0.543 & 0.298 & 1.835 & 0.073 & -0.051 to 1.138 \\
\hline
\end{tabular}

${ }^{\mathrm{a}}$, no other diseases are used as a reference. ${ }^{*}, \mathrm{P}$ values indicate that the independent variable has a statistical significance on the dependent variable in the regression equation.

in the portal area (16). However, liver fibrosis may progress even after surgery (17), especially with recurring cholangitis which causes continuous damage to the liver (18). Studies have shown that about $60 \%$ of children still require liver transplantation before the age of 20 (19-21), and some studies have also shown that patients undergoing liver transplantation have a higher long-term survival rate than those undergoing Kasai surgery (22). Increasingly, families of children with BA have realized that eventually liver transplantation may be required and has high expectations for liver transplantation. Unfortunately, at present, there is a lack of readily available information regarding pediatric liver transplantation. This lack of knowledge can cause anxiety in caregivers, which indirectly translates to a decline in the children's quality of life. This study confirmed that the caregiver's knowledge of liver transplantation affects the quality of life of the child. Therefore, medical staff should take the initiative to provide relevant knowledge about liver transplantation and answer the questions of caregivers of children with BA. At the same time, the writing of popular science books on pediatric liver transplantation and the popularization of pediatric liver transplantation scientific knowledge should be strengthened.

Studies have found that the method of payment of medical expenses is related to the social living standard and quality of life of children after liver transplantation, and children with medical insurance have a higher quality of life than children without medical insurance $(23,24)$. However, no similar reports have been found in children with BA. As $\mathrm{BA}$ is also a disease that exerts a huge financial burden on families, the method of payment of medical expenses may be related to the quality of life of these children. Unexpectedly, multiple linear regression analysis revealed that the payment method of medical expenses and the monthly medical costs incurred did not affect the quality of life of children with BA. It is possible that with improvements in China's social security system, the vast majority of rural and urban residents can access a basic social medical insurance or 
other subsidies. In this survey, the hospitalization expenses were fully self-funded in only $18.57 \%$ of children, with the remainder of children being funded by medical insurance or other subsidies. Therefore, families with reasonable medical insurance will experience less financial burden, which translates to improved quality of life for the sick child.

Cholangitis is the most common complication following Kasai surgery, with a reported incidence of $30-60 \%$, and it also affects a considerable number of adult patients $(7,25,26)$. Patients with more frequent and severe the postoperative cholangitis will experience poorer bile drainage, more obvious itching and nutritional deficiencies, and rapidly deteriorating liver fibrosis (27-29). Moreover, studies have shown that there is a correlation between the quality of life of children undergoing liver transplantation and the occurrence of postoperative complications (30). Therefore, one might speculate, that recurrent cholangitis may have a significant impact on the quality of life in children, there is a lack of literature to support this. The current study examined the incidence of cholangitis in children with BA, however, there was no correlation between the incidence of cholangitis and the quality of life of these children. This may be related to the establishment of a comprehensive follow-up system after BA surgery in the hospital, including an online booking system for postoperative follow-up appointments. Furthermore, postoperative management is conducted by a multi-disciplinary team which includes surgeons, clinical pharmacists, and nursing experts. All this facilitates the early detection and treatment of cholangitis in these children, thereby relieving caregiver stress and improving the quality of life of the children.

This study also investigated the emotional feelings of caregivers of BA children and found that only $27.1 \%$ had no particular emotional feelings. Nearly half of the caregivers felt tired and depressed. As the emotional response of caregivers can be transmitted to children through longterm daily contact, medical staff should pay attention to the mental well-being of caregivers and provide psychological counseling and support if required, so as to avoid negatively impacting the physical and mental health of the children.

This study had certain limitations. The cross-sectional survey design could not provide sufficient information on changes in the quality of life. Future studies should lengthen the follow-up period to investigate the changes in the quality of life of the same population over 1, 2, 3, and 5 years. Furthermore, the impact of liver transplantation on the quality of life of children with BA should be assessed.

\section{Conclusions}

This study demonstrated that the quality of life of BA children after surgery is significantly lower than that of agematched healthy children. The main influencing factors on the quality of life are co-existing diseases, the caregiver's understanding level of liver transplantation, and the caregiver's emotional coping ability. Thus, there should be a particular focus on BA children with co-existing diseases by providing accessible treatment and nursing care. It is also vitally important to provide knowledge and emotional support to caregivers so as to prevent their stress exerting a negative impact on the quality of life of BA children after surgery.

\section{Acknowledgments}

Funding: None.

\section{Footnote}

Reporting Checklist: The authors have completed the SURGE reporting checklist. Available at https://dx.doi. org/10.21037/tp-21-391

Data Sharing Statement: Available at https://dx.doi. org/10.21037/tp-21-391

Conflicts of Interest: All authors have completed the ICMJE uniform disclosure form (available at https://dx.doi. org/10.21037/tp-21-391). The authors have no conflicts of interest to declare.

Ethical Statement: The authors are accountable for all aspects of the work in ensuring that questions related to the accuracy or integrity of any part of the work are appropriately investigated and resolved. All procedures performed in this study involving human participants were in accordance with the Declaration of Helsinki (as revised in 2013). The study was approved by the Biomedical Ethics Committee of the West China Hospital, Sichuan University, China (\#20191082) and informed consent was taken from parents of all the patients.

Disclaimer: The views expressed in the submitted article are the authors' own opinions.

Open Access Statement: This is an Open Access article 
distributed in accordance with the Creative Commons Attribution-NonCommercial-NoDerivs 4.0 International License (CC BY-NC-ND 4.0), which permits the noncommercial replication and distribution of the article with the strict proviso that no changes or edits are made and the original work is properly cited (including links to both the formal publication through the relevant DOI and the license). See: https://creativecommons.org/licenses/by-nc-nd/4.0/.

\section{References}

1. Wang G, Feng JX. Pediatric Abdominal Surgery. Beijing: People's Medical Publishing House, 2011.

2. Shi CW. Pediatric Surgery, 4th. Beijing: People's Medical Publishing House, 2012.

3. Zhan JH, Wang L. Current status and prospects of pediatric hepatobiliary surgery. J Clin Surg 2017;25:890-1.

4. Lind RC, de Vries W, Keyzer CM, et al. Health status and quality of life in adult biliary atresia patients surviving with their native livers. Eur J Pediatr Surg 2015;25:e2.

5. Sundaram SS, Alonso EM, Haber B, et al. Health related quality of life in patients with biliary atresia surviving with their native liver. J Pediatr 2013;163:1052-7.e2.

6. Shinkai M, Ohhama Y, Take H, et al. Long-term outcome of children with biliary atresia who were not transplanted after the Kasai operation: >20-year experience at a children's hospital. J Pediatr Gastroenterol Nutr 2009;48:443-50.

7. $\mathrm{Ng} \mathrm{VL,} \mathrm{Haber} \mathrm{BH,} \mathrm{Magee} \mathrm{JC,} \mathrm{et} \mathrm{al.} \mathrm{Medical} \mathrm{status} \mathrm{of} 219$ children with biliary atresia surviving long-term with their native livers: results from a North American multicenter consortium. J Pediatr 2014;165:539-546.e2.

8. Lee WS, Ong SY. Health-Related Quality of Life in Children with Biliary Atresia Living with Native Livers. Ann Acad Med Singap 2016;45:61-8.

9. Varni JW, Seid M, Kurtin PS. PedsQL 4.0: reliability and validity of the Pediatric Quality of Life Inventory version 4.0 generic core scales in healthy and patient populations. Med Care 2001;39:800-12.

10. Gandek B, Ware JE, Aaronson NK, et al. Cross-validation of item selection and scoring for the SF-12 Health Survey in nine countries: results from the IQOLA Project. International Quality of Life Assessment. J Clin Epidemiol 1998;51:1171-8.

11. Lee PH, Wong FK, Wang SL, et al. Substitution of SF36 by SF-12 Among Hong Kong Chinese Older Adults: Secondary Analysis of Randomized Controlled Trials. Int J Behav Med 2016;23:635-44.
12. Castro Alves L, Kendall MC. Sample size and the establishment of safety in perioperative medicine. Actas Urol Esp (Engl Ed) 2018;42:610.

13. Chen W, Chen XA, Feng JX. Application of nutritional risk screening tools in patients with biliary atresia. J Clin Pediatr Surg 2015;14:68-70.

14. Alonso G, Duca P, Pasqualini T, et al. Evaluation of catchup growth after liver transplantation in children with biliary atresia. Pediatr Transplant 2004 Jun;8:255-9.

15. Nio $M$, Wada $M$, Sasaki H, et al. Technical standardization of Kasai portoenterostomy for biliary atresia. J Pediatr Surg 2016;51:2105-8.

16. Lampela H, Kosola S, Heikkilä $P$, et al. Native liver histology after successful portoenterostomy in biliary atresia. J Clin Gastroenterol 2014;48:721-8.

17. Medappil N, Jacob M, Lochan R, et al. Kasai portoenterostomy for biliary atresia - Surgical precautions for better outcomes. J Pediatr Surg 2019;54:868-9.

18. Song TT, Zhan JH, Gao W, et al. Changes of hepatic pathology in children with biliary atresia after Kasai portoenterstomy. Chin J Pediatr Surg 2014;35:603-7.

19. Nizery L, Chardot C, Sissaoui S, et al. Biliary atresia: Clinical advances and perspectives. Clin Res Hepatol Gastroenterol 2016;40:281-7.

20. Zhou RJ, Ming AX, Diao M, et al. The short-and mid-term prognosis and influencing factors of Kasai portoenterostomy for 220 cases of type III biliary atresia. Chin J Gen Surg 2019;(8):659-62.

21. Yu L, Guo Y, Ke HJ, et al. Laparoscopic portoenterostomy versus open portoenterostomy for children with biliary atresia :meta-analysis of comparative studies. Chin J Pediatr Surg 2019;(7):613-21.

22. LeeVan E, Matsuoka L, Cao S, et al. Biliary-Enteric Drainage vs Primary Liver Transplant as Initial Treatment for Children With Biliary Atresia. JAMA Surg 2019;154:26-32.

23. Tang Y, Liu Y, Wang ZJ, et al. Applicatin of individual intervene model in the following-up of recipient after liver transplantation. J Clin Pediatr Surg 2016;15:41-5.

24. Gao F, Lu YF. Survey of quality of life among the pediatric patients who have received the liver transplantation for one year. Chin J Prac Nurs 2019;35:1256-60.

25. Bijl EJ, Bharwani KD, Houwen RH, et al. The long-term outcome of the Kasai operation in patients with biliary atresia: a systematic review. Neth J Med 2013;71:170-3.

26. Kumagi T, Drenth JP, Guttman O, et al. Biliary atresia and survival into adulthood without transplantation: a collaborative multicentre clinic review. Liver Int 
2012;32:510-8.

27. Cheng K, Molleston JP, Bennett WE Jr. Cholangitis in Patients With Biliary Atresia Receiving Hepatoportoenterostomy: A National Database Study. J Pediatr Gastroenterol Nutr 2020;71:452-8.

28. Tarro TM, Song L, Carter BA, et al. Prophylactic Antibiotics Did Not Decrease Recurrent Cholangitis in Patients with Biliary Atresia After Kasai Portoenterostomy. Open Forum Infect Dis 2020;7:S354-S355.

Cite this article as: Liang Y, Yu H, Shu F, Huang W, Jiang X, $\mathrm{Xu}$ Z, Zhang T, Xiang B, Jin S. Factors influencing the quality of life in children after biliary atresia treatment. Transl Pediatr 2021;10(10):2496-2505. doi: 10.21037/tp-21-391
29. Hung PY, Chen CC, Chen WJ, et al. Long-term prognosis of patients with biliary atresia: a 25 year summary. $\mathrm{J}$ Pediatr Gastroenterol Nutr 2006;42:190-5.

30. Yang Y, Xin WQ, Xiang B, et al. Quality of life of pediatric living donor liver transplantation recipient and it's influencing factors. Chin J Bas Clin Gen Surg 2018,25:912-6.

(English Language Editor: J. Teoh) 